\title{
On Symbolic Dynamics of One-Humped Maps of the Interval
}

\author{
Peter Grassberger \\ Physics Department, University of Wuppertal, Wuppertal, West-Germany \\ Z. Naturforsch. 43a, 671-680 (1988); received May 16, 1988
}

\begin{abstract}
We present an explicit construction of minimal deterministic automata which accept the languages of L-R symbolic sequences of unimodal maps resp. arbitrarily close approximations thereof. They are used to study a recently introduced complexity measure of this language which we conjecture to be a new invariant under diffeomorphisms. On each graph corresponding to such an automaton, the evolution is a topological Markov chain which does not seem to correspond to a partition of the interval into a countable number of intervals.
\end{abstract}

\section{Introduction}

One of the most interesting discoveries of the last decades is that formally deterministic but "chaotic" systems can behave in a way which only can be described as random.

In particular, there occurs an information loss during the evolution of the system. If it is not permanently observed, any prediction can only be made with a precision worse than the precision with which the initial conditions were known. If one keeps to some fixed precision by making a finite partition of phase space, the motion on this coarse-grained phase space is strictly random. The information loss per unit of time - the Kolmogorov-Sinai metric entropy [1] - is then the same for all sufficiently fine or otherwise suitably chosen partitions. The latter are called "generating partitions".

Assume that one has a discrete time system, and assume that the phase space point representing the system at time $t$ is in the $i$-th element of the partition: $s_{t}=i$. Then any orbit can be encoded as a string $\left(\ldots s_{t}\right.$, $\left.s_{t+1}, s_{t+2}, \ldots\right)$ called a symbol sequence. This encoding is one-to-one up to sets of points of measure zero. It is called symbolic dynamics [2].

Take for instance one-humped ("unimodal") maps $x \rightarrow F(x)$ of an interval onto itself (in addition, we assume some further properties [2] which imply in particular that $F$ has a unique attractor). A prototype is the quadratic ("logistic") map

$$
x_{n+1}=a-x_{n}^{2} .
$$

Its generating partition is a binary partition into points right or left of the critical point which we put

Reprint requests to Prof. Dr. P. Grassberger, Fachbereich 8, Naturwissenschaften I, Bergische Universität, Gesamthochschule Wuppertal, Gauss-Straße 20, Postfach 100127 , D-5600 Wuppertal 1. at $x=0$ also for other unimodal maps. Denote

$$
s_{n}=\left\{\begin{array}{lll}
\mathrm{L} & \text { if } & x_{n}<0, \\
\mathrm{C} & \text { if } & x_{n}=0, \\
\mathrm{R} & \text { if } & x_{n}>0 .
\end{array}\right.
$$

Since nearly all (in the sense of Lebesgue measure) trajectories will avoid the point $x=0$, they can be encoded as sequences of R's and L's only. The Shannon information stored in such a sequence is proportional to its length, with the proportionality constant being the Kolmogorov entropy.

Strictly spoken, the Kolmogorov entropy is the information density only for one particular time invariant distribution, the so-called natural or BowenRuelle-Sinai [1] measure. Chaotic systems allow for infinitely many invariant distributions. But all except the natural one are unstable in the sense that the density of the natural measure is the (weak) limit $\varrho(x)=\lim _{N \rightarrow \infty} N^{-1} \sum_{i=1}^{N} \delta\left(x-x_{n}\right)$, for nearly all start values $x_{0}$ and/or when arbitrarily small noise is added. The entropy depends in general on the distribution chosen.

A priori independent of any measure is the "topological entropy". It is related to the concept of a "topological Markov chain". Just as a (first order) Markov chain is a process where probabilities show no memory effects, in a topological Markov process the set of all sequences involves no memory: any sequence of symbols $\left\{s_{n}\right\}$ is allowed which contains only allowed transitions $S_{n} \rightarrow S_{n+1}$. A generating partition is called Markovian if the evolution on its elements is a topological Markov process. The topological entropy is an upper bound for the entropies of all invariant measures and is indeed attained for one measure (the socalled maximum entropy measure). Technically, Mar- 
kov partitions for one-humped maps are defined as follows [3]: a partition is Markov if the image under $F$ of any of its elements is again an element or a union of elements. In this way, it is guaranteed that for any two elements $i$ and $j$ of the partition a preimage in $j$ exists for a point $x$ in $i$ iff $j$ is mapped onto $i$, independently of the precise point $x$.

The importance of Markov partitions is that they allow to associate topological (and for maximum entropy measures also conventional) Markov processes to the evolution of chaotic systems. We shall see in Sect. 4 that the evolution can be explicitely associated to a Markov process also without having an explicit Markov partition in this sense.

Randomness is not the most fascinating feature of chaotic systems. Much more interesting are the very rich structures which are found for most such systems. This concerns first of all the way how the behaviour depends on control parameters. Very well studied in this context are again one-humped maps. Changing the constant a in (1), one finds an extremely rich and in a certain sense universal pattern of chaotic attactors alternating with periodic "windows", with perioddoubling cascades inside these windows, etc. [2].

Another manifestation of the complexity of chaotic systems is seen in the geometry of the orbits. As examples we just mention Julia sets [4] and Hamiltonian systems with intermixed chaotic and regular domains [5].

Finally, the complex structure of chaotic dynamical systems is reflected also in the structure of the possible symbol sequences. For any dynamical system with a finite generating partition the set of all possible symbol sequences can be considered as a formal language. The randomness of the system corresponds to the fact that nearly each individual sequence has a positive complexity in the sense of Kolmogorov [6]. The structures mentioned above correspond to the fact that in general also the language is complex: not only each individual sequence can be hard to describe but also the ensemble of all sequences can be very hard to describe.

That these two need not be the same is most easily seen for the logistic map. For $a=2$, all symbol sequences are allowed and the natural measure corresponds to equipartition in the space of symbol sequences. Thus the language is completely trivial to

* Another exception is that no invariant measure on the graph exists although the relative frequencies of the nodes do not vanish. Such cases do exist (G. Keller, private communication) but are neglected in the following. describe, while any particular sequence of length $N$ cannot be compressed to less then $N$ bits. Take now the Feigenbaum point [7] $a=1.401155 \ldots$. There, the metric entropy is zero since a sequence of length $N$ carries only $\approx \log N$ bits of information [8]. But any description of the set of all allowed L-R sequences needs infinitely many bits, just due to the long-range correlations manifested in the logarithmic increase of information.

When speaking of the complexity of a formal language, one usually means the complexity of its "grammar" [9]. This means, one asks only for the difficulty of describing the set of all sequences, disregarding completely any probabilistic aspects. But it is obvious that probabilities can influence very strongly whether we naively consider a language as complex or not. Two authors may use the identical set of words and grammatical rules; if one of them uses frequently only a small subset then we consider his language certainly less complex.

In [8], we have introduced several quantities which in one way or the other measure this complexity. In particular, we introduced the "set complexity" SC as follows. Assume we want to check if any given string belongs to the set (i.e. is grammatically correct). In the simplest case of "regular" grammars, this can be done by means of a finite deterministic automaton [9]. This is a directed graph, with each link labelled by one of the "letters of the alphabet" of the strings (i.e. by L or $\mathrm{R}$ for one-humped maps). One of the nodes is labelled "start". For each letter, there is at most one link leaving any node. Scanning the string for correctness corresponds then to moving on the graph from node to node, starting from the node labelled "start". Assume the string has been checked up to and including $s_{n}$, and that this check has led us to node $i$. The $(n+1)$-st symbol $s_{n+1}$ is correct iff there is a (necessarily unique) link labelled " $s_{n+1}$ " leaving this node. If so, we follow $i$, reaching thus some other node $k$. From there we continue the check with symbol $s_{n+2}$.

Since we consider only stationary ensembles (invariant measures), this procedure defines a measure on the nodes of the graph, with probability $p_{i}$ to visit the $i$-th node (except when nearly all symbol sequences correspond to non-recurrent walks on the graph*; then we put $p_{i}=0$ and $\mathrm{SC}=\infty$; see the case of Feigenbaum points, Sect. $3 \mathrm{~d}$ ). The set complexity is defined as

$$
\mathrm{SC}=\underset{\text { graphs }}{\min }\left\{-\sum_{i} p_{i} \log p_{i}\right\},
$$


where the minimum is over all graphs which accept all correct strings and only these. It is the minimal average Shannon information which has to be stored if we want to scan a typical sequence for correctness.

Among commonly studied definitions of complexity, the one most closely related to the above is that of a regular grammar [10]: the logarithm of the number of nodes of the smallest associated graph. This is also the maximal information which has to be stored at any time during a scan, if no invariant measure on the graph is given. If such a measure is naturally defined as in our case, it seems clear that the Shannon information is more relevant, just as the Kolmogorov entropy is usually of greater interest than the topological entropy.

Furthermore, and this is of crucial importance for us, while the regular grammar complexity of [10] applies only to finite graphs (i.e. regular languages), the above construction obviously works also for infinite graphs. Thus we can define with it also complexities of non-regular languages equiped with invariant measures, as will be done below.

In the next section, we shall construct explicitly the graphs of automata accepting the R-L sequences of any one-humped map. They are finite in the case of periodic "windows", i.e. when the attractor is periodic, and at band-merging ("Misiurewicz" or "crisis" [11]) points. Otherwise they are infinite. They seem to be minimal in the sense of (3). When they are infinite, we construct systematic and arbitrarily close approximations which accept all those L-R sequences which have correct subsequences up to a given length.

These automata are a tool to study the complexity of the ensemble of symbol sequences of e.g. the logistic map. This will be done in Sect. 3 for the natural measure.

We shall see that the SC is finite for nearly all parameters $a$. It is infinite only for Feigenbaum points, and it diverges when we approach tangent bifurcation points (i.e. points where new periodic windows open up) from below. This is directly connected with the long time correlations at these points. It indicates that the SC is indeed a useful measure of complexity.

\section{The Algorithm}

Before describing the automata construction, we have to present some more well known facts $[2,12]$.
First, the "kneading sequence" $K$ of $F$ is the symbol sequence obtained by starting with the image of the critical point, $x_{1}=F(0)$. It always starts $K=\mathrm{R} \ldots$. For superstable periodic orbits (i.e. orbits which contain $x_{0}$ ), $K$ contains infinitely many " $C$ "'s. Otherwise, it contains none. We shall denote by $K^{\prime}$ the sequence obtained from $K$ by dropping the leading " $\mathrm{R}$ ".

Next, define for any sequence $S=\left(s_{1}, s_{2}, \ldots\right)$ a sequence $\tau(S)=\left(t_{1}, t_{2}, \ldots\right)$ as follows: $t_{n}=0$ if the number of "R"'s in $S$ up to (and including) position $n$ is even, and $t_{n}=1$ if this number is odd. Denote the Bernoulli shift as $\sigma,(S)_{i}=s_{i+1}$, and denote by $\overline{\tau(S)}$ the sequence obtained from $\tau(S)$ by exchanging 0 and 1 . Finally, order all $0-1$ sequences by considering them as binary representations of numbers from $[0,1]$.

Then it is known that any $S$ is an allowed symbol sequence for a one-humped map if and only if one has [12]

$$
\overline{\tau(K)} \leq \sigma^{m}(\tau(S)) \leq \tau(K) \text { for all } m \geq 0 .
$$

(Except for a possible prefix consisting of arbitrarily many " $L$ "'s, preceeded eventually by a single "R". The latter occurs if we start with $x_{0}$ near the negative unstable fixed point or with $x_{0}>F(0)$. We shall exclude this in the following by considering only start values $x_{0}$ in the interval $\left[F^{2}(0), F(0)\right]$.)

This means that the set of allowed symbol strings can be characterized by an (in general infinite) list of forbidden "words". Take e.g. (1) with $a=1.8$. There, the kneading sequence is

$$
K=\text { RLLRLRRLRRRLRLR ... }
$$

such that

$$
K^{\prime} \quad=\text { LLRLRRLRRRL } \ldots
$$

and

$$
\tau(K)=111001001011001 \ldots
$$

The first forbidden words in $\tau(S)$ are 1111 and 0000 , corresponding to the forbidden word LLL in $S$. The next forbidden words in $S$ are LLRR, LLRLRL, LLRLRRR, LLRLRRLRL, etc. The rule by which these words are formed is very simple. First, there is at most one forbidden word for each length $N$. More precisely, there is a forbidden word of length $N$ in $S$ iff $\tau(K)$ contains a " 0 " at the $(N+1)$-st position, and this word is obtained from the first $N$ symbols of $K^{\prime}$ by changing its last symbol.

Assume there is a forbidden word of length $N$. Let us denote then by $G_{N}$ the formal language consisting of all sequences containing no forbidden words of 


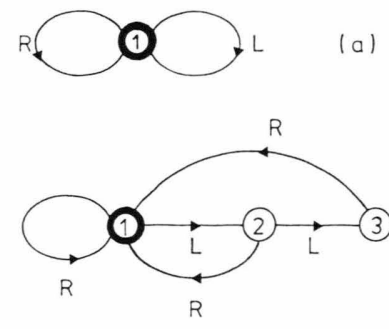

(b)

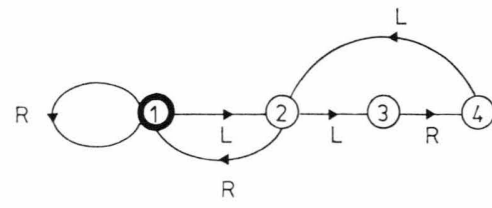

(c)

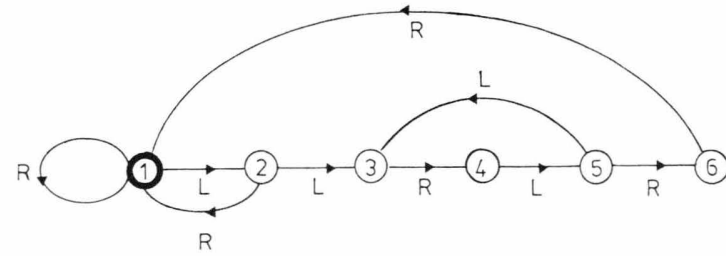

Fig. 1. Finite automata accepting the first approximations $G_{1}$ to the set of symbol sequences at $a=1.8$ with $l=0$ (Fig. $1 \mathrm{a}$ ), $l=3$ (Fig. $1 \mathrm{~b}$ ), $l=4$ (Fig. $1 \mathrm{c}$ ), and $l=6$ (Figure $1 \mathrm{~d}$ ). Here and in all following graphs, the heavy node is the start.

legth $\leq N$. For increasing $N$, these form better and better approximations of the set of all allowed sequences, but for all finite $N$ they are regular languages.

This simple structure can be used to construct recursively a sequence of automata accepting the languages $D_{N}$. In a periodic window, this construction stops at a finite $N$. Otherwise, it gives finally an infinite graph. In general, this is the desired result. Only at band-merging points, where the language is regular but not a subshift of finite type [13], we have to replace it at the end by a simpler graph.

We start the recursion with a graph consisting simply of a single node and two loops labelled " $L$ " and "R" (Figure $1 \mathrm{a}$ ). It accepts all sequences.

After a finite number of steps, the graph contains $N$ nodes and accepts the language $G_{N}$. The start is at node 1. For each $i<N, i \geq 1$, there is one link connecting node $i$ to node $i+1$. It is labelled by the $i$-th symbol of $K^{\prime}$. From the $N$-th node there leaves only one link labelled by the $N$-th symbol of $K^{\prime}$. Finally, there are further links determined by the recursion.

This recursion stops at $N$ if there is no forbidden word longer then $N$. Otherwise, call $N^{\prime}$ the length of the next forbidden word. In the step $N \rightarrow N^{\prime}$, we add
$N^{\prime}-N$ new nodes, label the links between successive nodes as above, cancel the previous link starting from node $N$, and add links leading from the new nodes to the old ones as follows: for every path in the old graph starting at node 1 and consisting of $m$ symbols of $K^{\prime}\left(N<m<N^{\prime}\right)$ followed by one symbol not in $K^{\prime}$, we add one link such that the same path exists also in the new graph and leads to the same node as in the old graph. Finally, we add one link leaving the last node such that the path consisting of the $N^{\prime}$ first symbols of $K^{\prime}$ leads to the same node as in the old graph.

This construction might sound complicated but is indeed very simple. The first few graphs obtained for $a=1.8$ are shown in Figure 1. On a computer, the size of the largest graph obtainable is limited only by round-off errors in the iteration of (1) which typically prevent getting more than $\sim$ hundred significant symbols of $K$. A similar construction of finite approximate grammars was given in [14]. The point to be noticed is not that finite approximations recognizing forbidden words up to any given length $N$ can be given, but that their graph size is only $N$ (a priori, the size could have been anywhere between $N$ and $2^{N-1}$ [10]). This was only possible by using the fact that a start node exists. Start nodes are usually not used in dynamical systems, while they are important in formal language theory.

\section{Special Cases}

We shall now see what this construction yields in the special cases of periodic windows, band-merging points, tangent bifurcation points, and Feigenbaum points.

\section{a) Periodic Windows}

In periodic windows, the kneading sequence is periodic. In each window of period $p$, there is one superstable point a, where the orbit contains the critical point $x=0$. At the end of each period- $p$ interval, there is a bifurcation point followed by an interval with period $2 p$. The kneading sequence does not change at the bifurcation points but rather at the superstable points. On both sides of $a_{s}$, the graphs are thus different: below $a_{s}$, the graph contains $p-1$ nodes, above it, it has $2 p-1$ nodes. Of the latter, $p-1$ nodes belong to a transient part which cannot be re-entered once it has been left. For all periods obtained by period doubling, the graph contains a transient part also 

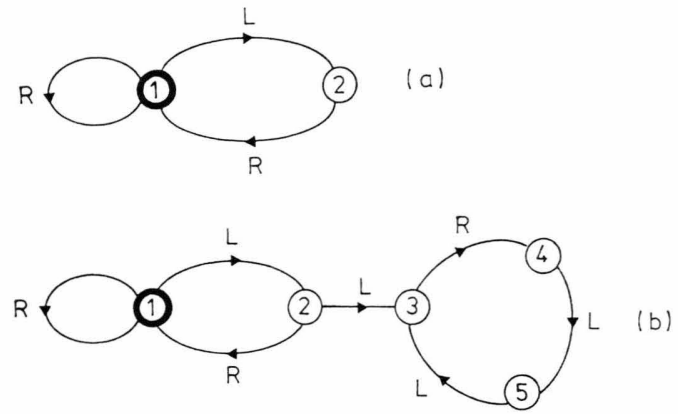

Fig. 2. Minimal graphs accepting the symbol sequences in the second period 3 window: a) below the superstable point; b) above it. - The latter is also the graph in the following period 6 window up to its superstable point.
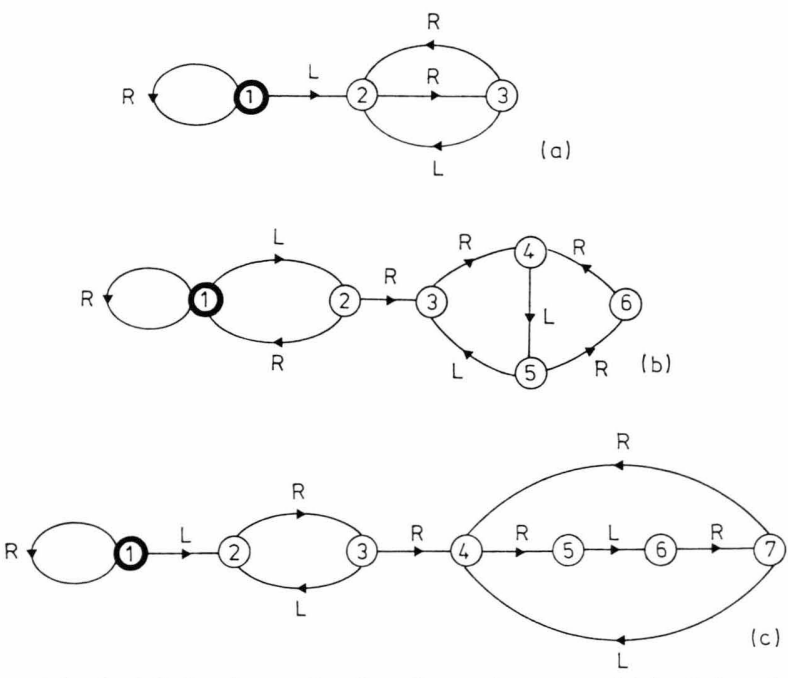

Fig. 3. Minimal graphs for the points at which 2 bands merge into 1 (Fig. 3 a), 3 into 1 (Fig. 3 b), and at the first point where 4 merge into 2 (Figure $3 \mathrm{c}$ ).

below $a_{s}$. As examples, we present in Fig. 2 the graphs for the period 3 window.

The natural measure is concentrated on the periodic orbit. Thus, the transient parts of these graphs have zero weight. Its $\mathrm{SC}$ in a window of is always exactly computable and is $\leq \log$ (period).

\section{b) Band-Merging Points}

At a band-merging point, the kneading sequence also has a periodic tail but there are infinitely many forbidden words, whence our construction gives an infinite graph. This is however not the minimal graph. Finite graphs are obtained by identifying nodes as described e.g. in [9]. As examples we show in Fig. 3 the minimal graphs at the mergings $2 \rightarrow 1,3 \rightarrow 1$, and at the first merging $4 \rightarrow 2$. Figure $3 \mathrm{a}$ accepts all sequences not containing LL, LRRL, LRRRRL, etc. which we write $\mathrm{L}(\mathrm{RR}){ }^{*} \mathrm{~L}$. Similarly, Fig. 3 b corresponds to $\mathrm{LL}(\mathrm{RLR}) * \mathrm{RR}$ and $\mathrm{LL}(\mathrm{RLR}){ }^{*} \mathrm{~L}$ being forbidden, while Fig. $3 \mathrm{c}$ forbids LL, LRRL, and LRRR(LRLR)*R.

All these graphs contain transient parts which carry zero natural measure. In Figs. $3 \mathrm{a}$ and $3 \mathrm{~b}$, they are different from those in the periodic windows insofar as there are no corresponding transient regions in $x$. They are necessary only since without them the start node would not be unique. The weights of the other nodes cannot be given in closed form but can be estimated easily from simulations. The SC is obviously finite at band-merging points.

When approaching a band-merging point $a_{\mathrm{c}}$ either from above or below, longer and longer parts of the kneading sequence agree with that at $a_{\mathrm{c}}$. This means that part of the graph close to the start is identical to that of the infinite graph at $a_{\mathrm{c}}$ before the minimization. Also, the weights of the nodes converge to the weights of the non-minimized graph. This means that the SC is discontinuous at $a_{\mathrm{c}}$, with the limits from both sides existing and agreeing with each other but not with the value at $a_{\mathrm{c}}$. This was also checked numerically.

\section{c) Tangent Bifurcation Points}

These are the points where periodic windows start. Exactly at these points, the graph is the same as in the periodic window, the only difference being in the slower convergence of an arbitrary measure towards the natural one.

Below these points, we observe intermittency [15]. More precisely, call $a_{\mathrm{c}}$ the bifurcation point and $a_{\mathrm{c}}-a=\varepsilon$. Then the typical length of laminar periods is $T \sim 1 / \sqrt{\varepsilon}$. We expect that this is also the typical number of nodes which have non-negligible weight, whence $\mathrm{SC} \sim \log T \sim \frac{1}{2} \log \varepsilon$. This was indeed verified numerically below the opening of the period 3 window.

\section{d) Feigenbaum Points}

By this we mean the accumulation points of subharmonic bifurcations. the most prominent one is the accumulation point of bifurcations $2^{k} \rightarrow 2^{k+1}$ which for (1) occurs at $a_{x}=1.401155 \ldots$. 


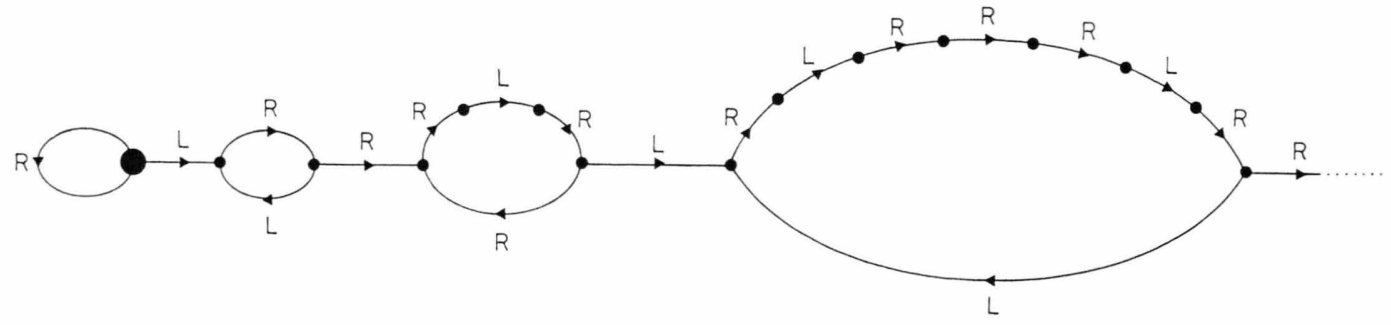

Fig. 4. Part of the graph at the Feigenbaum point (first accumulation point of period doublings).

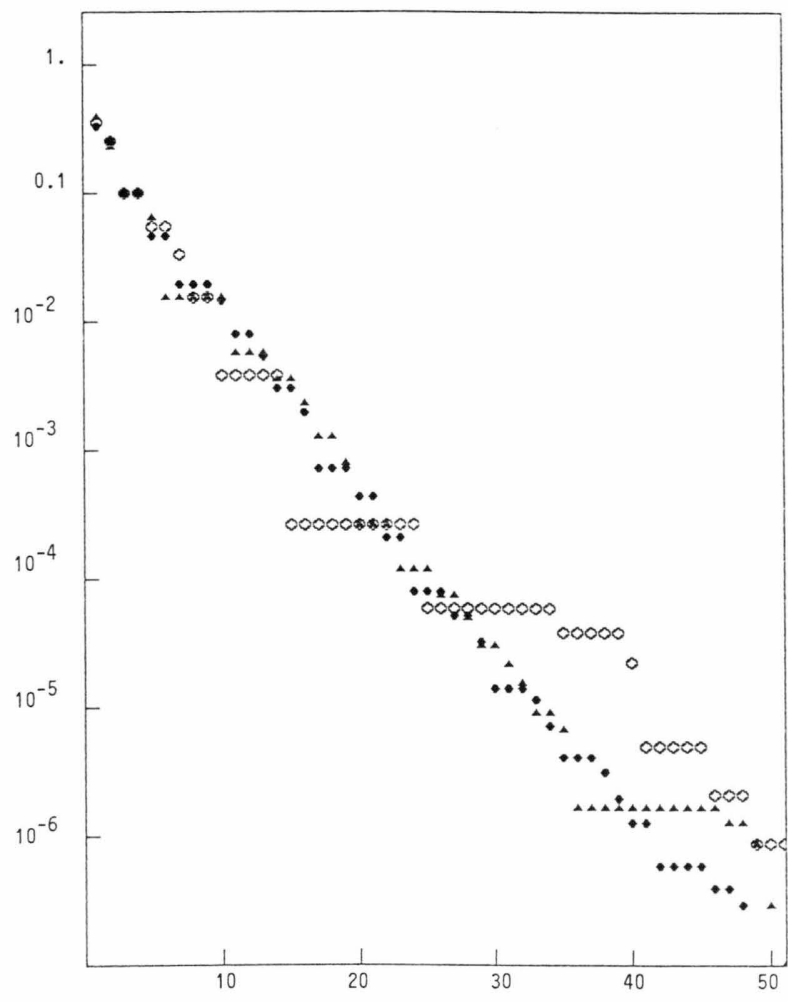

Fig. 5. Natural weights of nodes in the minimal graphs at $a=1.89(\mathbf{\square}), 1.9(\circ)$, and $1.91(\triangle)$.

At these points, the entropy is zero. This means that most sequences will look increasingly non-random, and thus for most nodes there will only be one leaving link. Furthermore, there are infinitely many transient domains in $x[16]$ which must also correspond to transient parts in the graphs. The resulting graph e.g. at $a_{\infty}$ is shown in Figure 4.

In the natural measure all transient domains have zero weight, whence it does not correspond to a normalizable measure on the graph of Figure 4. More precisely, the SC diverges when approaching $a_{\infty}$ both from above and below such that $\mathrm{SC} \sim k$ bits in a period $2^{k}$ band.

\section{e) Typical Chaotic Points}

The band-merging points are denumerable. Since it is known that the set of $a$-values with chaotic attractors has positive Lebesgue measure [17,18], it is clear that nearly all of them are not band-merging points. We conjecture that in all these cases there are indeed no finite graphs accepting just the set of all allowed sequences.

Thus, the set of all symbol sequences is not simple in this case, forming at least a context-free language [9]. But the natural measure could still be such that the SC is small. Numerically, we found that this is indeed the case. More precisely, the weights $p_{i}$ of the $i$-th node seem to decrease exponentially. This is demonstrated in Fig. 5 for 3 values of $a$.

Therefore, the SC converges very fast and can easily be estimated numerically. We show in Fig. 6 a the estimates obtained at $\sim 490$ points from $2 \times 10^{6}$ iterations each. We see clearly the divergence at tangent bifurcations and at Feigenbaum points, and the plateaus inside periodic windows. Just to locate these, we show in Fig. 6b the bifurcation diagram. Since tangent bifurcations and Feigenbaum points are dense in the chaotic region, the upward spikes in Fig. $6 \mathrm{a}$ should indeed be dense, just as the downward spikes are dense in a plot of Lyapunov exponents. The curve shown should thus be considered as a lower envelope.

\section{Topological Markov Chains and Markov Partitions}

Clearly, the walks on the graph induced by the map $F$ form a topological Markov chain. Furthermore, by specifying transition probabilities $p(i \rightarrow j)$ for going 


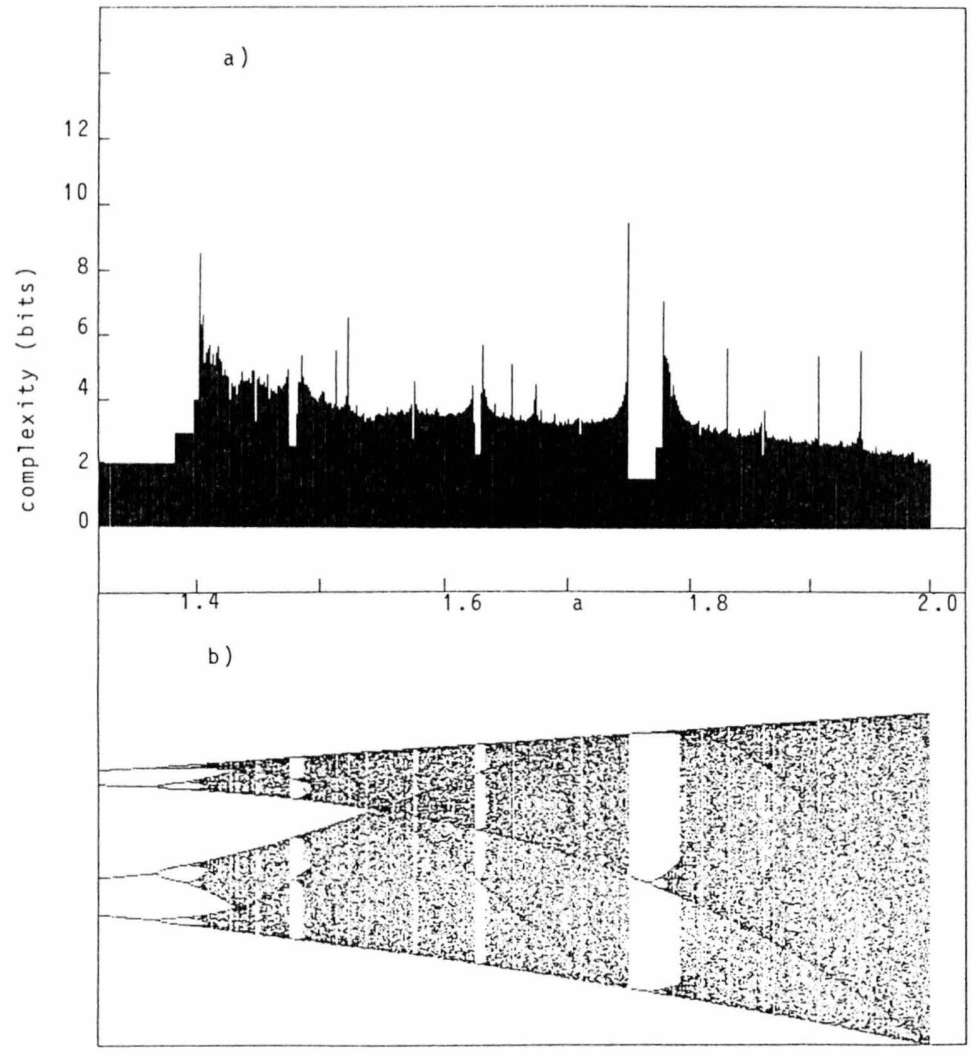

Fig. 6 .

Fig. 6. Set complexity according to the natural measure of the logistic map $x^{\prime}=F(x)=a-x^{2}$ versus the parameter $a$ (panel a) and bifurcation diagram (panel b).

Fig. 8. Partitions of the interval corresponding to Figs. $1 \mathrm{~b}$ to $1 \mathrm{~d}$. The heights of the curves are indicative of the density of the invariant measure. The shades of grey correspond to the links onto which the point $x$ maps under $\Phi_{3}$ (Fig. 7 a), $\Phi_{4}$ (Fig. 7 b), resp. $\Phi_{6}$ (Figure 7 c). The links starting at the start node corresponds to the lightest grey, those from the last node to the darkest.

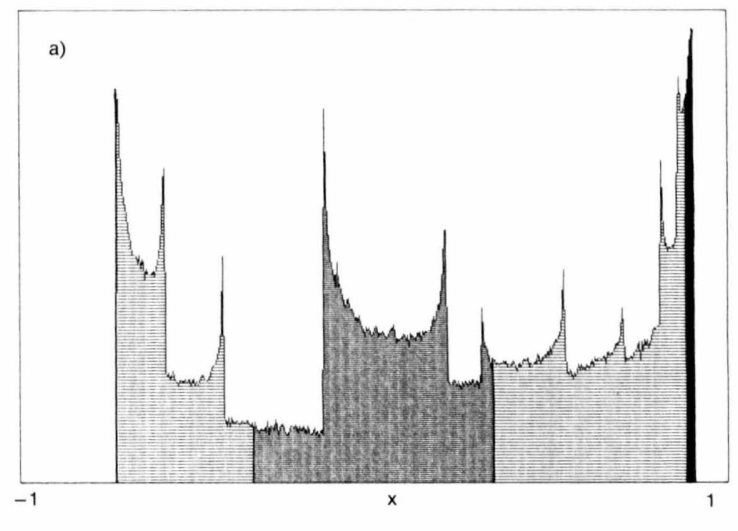

Fig. 8 a
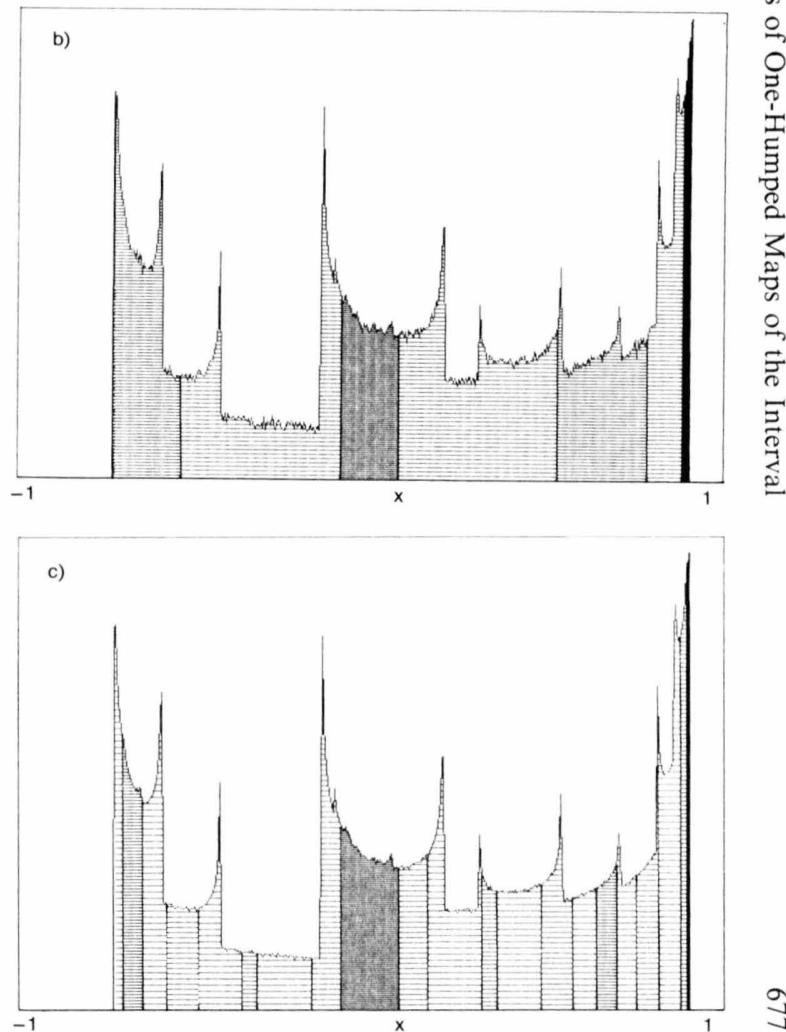


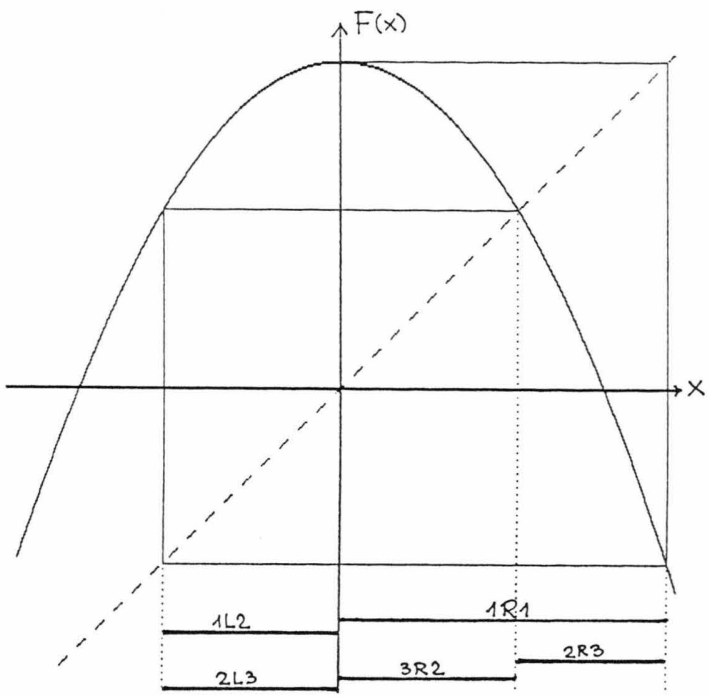

a)

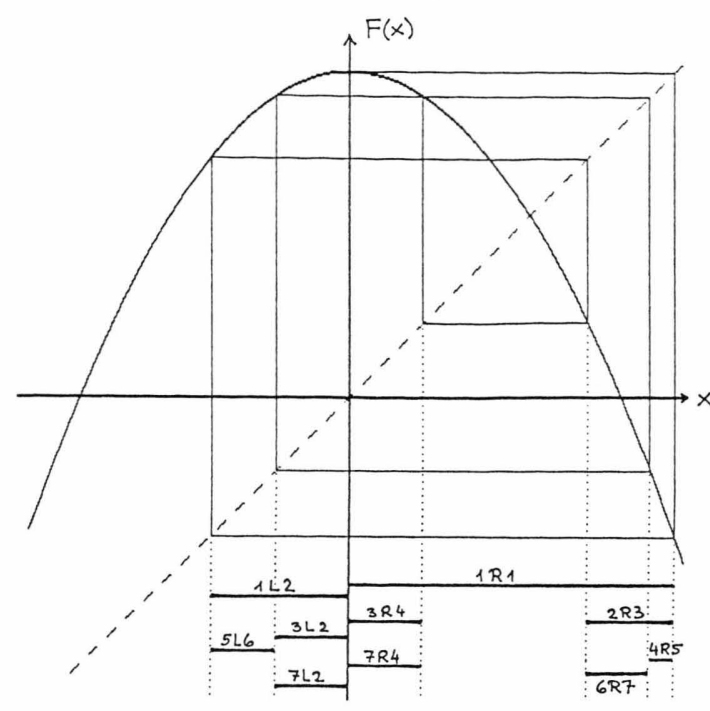

b)

Fig. 7. Intervals onto which the links of Figs $3 \mathrm{a}$, c are mapped under the map $\varphi$. Panels a resp. b correspond to panels a resp. c in Figure 3. By "1L2" we mean e.g. the link labelled "L" which leads from node 1 to node 2.

from link $i$ to link $j$, we define proper Markov processes. Notice that the process has to be defined over the links (not over the nodes). Otherwise, it would not be a faithful representation of the evolution of $F$ in cases such as Fig. $3 \mathrm{a}$ and $3 \mathrm{~b}$ where 2 nodes are connected by two different links.

Obviously, the entropy of any such process on a graph corresponding to a unimodal map $F$ cannot be larger than the topological entropy of $F$. On the other hand, the maximum entropy measure of $F$ corresponds to a stochastic process on the graph, and the process with maximal entropy on the graph must be Markovian. Thus, we have found that the evolution with the maximum entropy measure corresponds to a Markov process on the graph accepting the R-L sequences.

It seems thus suggestive that there should exist at least one map from the interval onto the links of the graph such that every link corresponds to an element of a generating partition. This would then be a Markov partition. We claim that this is not true in general if one requires a partition to correspond to a countable set of intervals. We see no way to map links bijectively onto intervals or even onto countable unions of intervals, except in periodic windows and at band-merging points. One may however generalize the concept of a partition such that each element is a Cantor set in which case there is no problem. Another possibility consists in partitioning not the interval but rather the space of itineraries. This was done in $[14,18]$.

There are two maps which rather naturally connect links of the graph to subsets of the interval $I=[F(F(0)), F(0)]$.

The first, let us call it $\psi$, is a map from each link $i$ onto an interval $I_{i} \subset I$. It is defined as follows:

(i) The two links emerging from the start node map onto the right resp. left half of the entire interval;

(ii) The interval $I_{i}$ corresponding to a link $i$ with label $s$ consists of all points with sign $s$ whose immediate preimages are in any of the intervals $I_{j}$ which correspond to the links joining up to link $i$.

For the first band-merging points (Fig. 3), these intervals are indicated in Fig. 7. We see in Fig. 7a that the intervals $I_{3}$ to $I_{5}$ form a partition of $I$. Since the first two links are transient, we can truncate them and find that the remainder defines indeed a Markov partition. The same is already not true in Fig. $7 \mathrm{~b}$ where truncation leads only to Markov partitions of subsets of $I$. In general, $\psi$ defines a topological Markov chain on a countable set of intervals, but these intervals form 
no partition of $I$ since they cover $I$ several times. In Fig. $7 \mathrm{~b}$ we could use all intersections of the $I_{i}$ 's as a partition, but in general the number of these intersections seems not countable.

The second map which we shall consider is always bijective but the images of links are in general neither intervals nor countable unions thereof. Take e.g. a map where there is a single band such that the evolution is mixing. Consider the following family of maps $\phi_{N}$ from the interval into the set of links: start with a random $x_{0}$ and move on the finite graph accepting $G_{N}$ according to the symbol sequence of its iterates, starting at the start node. To any $x_{i}$ associate the link crossed at the $(i+N-1)$-st move. Generalize this to all points $x$ by continuity (remember that we are considering the mixing case where the points $x_{i}$ are dense). The inverse of $\phi_{N}$ maps every link onto a finite union of intervals, and the map is indeed independent of $x_{0}$ for each $N$. The final map $\phi$ is obtained in the limit $N \rightarrow \infty$. But in this limit, the number of intervals corresponding to each link diverges exponentially, with finally each link mapping onto an everywhere dense Cantor set. For the graphs of Fig. 1, this is illustrated in Figure 8.

We thus conclude that we can associate in general the graphs either to "partitions" not made of intervals but of Cantor sets, or to sets of intervals which cover the entire interval more than once. We have not found any way to associate them to partitions into countable sets of intervals, although we cannot exclude that such partitions might exist.

Instead of using the graphs accepting the L-R symbol sequences, we could use any other associated topological Markov chain to define a SC. For each invariant measure $\mu$, the minimal SC over all possible Markov chains is clearly a topological invariant. We conjecture that at least for the natural measure this minimum is indeed obtained for the L-R partition, whence the SC computed in Sect. 3 is a new invariant under diffeomorphisms.

A further invariant consists in the minimal number of states of the topological Markov chain. This would

[1] J.-P. Eckmann and D. Ruelle, Rev. Mod. Phys. 57, 617 (1985).

[2] P. Collet and J.-P. Eckmann, Iterated Maps on the Interval as Dynamical Systems, Birkhauser, Boston 1980.

[3] R. Bowen, Amer. J. Math. 92, 725 (1970).

[4] H.-O. Peitgen and P. H. Richter, The Beauty of Fractals, Springer, Berlin 1986. be a generalization of what was called the "regular language complexity" of the grammar of symbol sequences in [10]. It is indeed a topological invariant but it seems less interesting in the present case since it is infinite for nearly all chaotic unimodal maps.

\section{Discussion}

Summarizing, we have found a simple explicit way to verify whether any binary symbol sequence is the L-R sequence of a given one-humped map. The "difficulty" of this scan, measured in terms of the average information about the past part of the sequence which has to be stored for this, is the SC. It is a complexity measure of the set of these sequences, taking into account the actual distribution of subsequences. Due to the latter, it was finite even in cases where purely algorithmic methods could not provide a finite measure of complexy. It is conjectured to be indeed the minimal such information over all possible partitions, and thus to be a new invariant under diffeomorphisms.

This scan was provided by an (in general infinite) graph such that the evolution corresponded to a motion on the graph. This motion is a topological Markov process which in general corresponds to a "partition" of the interval into a countable set of Cantor sets. We suggest that also for other non-hyperbolic systems the only Markov partitions are similarly complicated, though the topological Markov chains per se might be quite simple.

An important technical point was the fact that the graph representing the Markov chain had a start node. Only due to this, the association between the map and the Markov chain was unique.

I am very much indebted to $T$. von der Twer for interesting discussions and for carefully reading the manuscript, and to P. Collet for a very helpful discussion.

[5] A. J. Lichtenberg and M. A. Liebermann, Regular and Stochastic Motion Springer, New York 1983.

[6] V. M. Alekseev and M. V. Yakobson, Phys. Rep. 75, 287 (1981).

[7] M. Feigenbaum, J. Stat. Phys. 19, 25 (1978); 21, 669 (1979).

[8] P. Grassberger, Int. J. Theor. Phys. 25, 939 (1986). 
[9] J. E. Hopcroft and J. D. Ullman, Introduction to Automata Theory, Languages, and Computation, AddisonWesley, New York 1979.

[10] S. Wolfram, Commun. Math. Phs. 96, 15 (1984).

[11] S. Thomae and S. Grossmann, J. Stat. Phys. 26, 485 (1981); C. Grebogi, E. Ott, and J. A. Yorke, Physica 7D, 181 (1983).

[12] J.-P. Allouche and M. Cosnard, Grenoble preprint (1984); J. Dias de Deus et al., Lisbon preprint (1984).

[13] P. Billingsley, Ergodic Theory and Information, Wiley, New York 1965.
[14] F. Hofbauer, Israel J. Math. 34, 213 (1979); 38, 107 (1981); Erg. Theor. Dynam. Syst. 5, 237 (1985).

[15] Y. Pomeau and P. Manneville, Commun. Math. Phys. 74, 189 (1980).

[16] P. Grassberger and M. Scheunert, J. Stat. Phys. 26, 697 (1981).

[17] M. Misiurewicz, Publ. I. H. E. S. 53, 17 (1981); M. V. Yakobson, Commun. Math. Phys. 81, 39 (1981).

[18] P. Collet, preprint (1986). 